\title{
Near-infrared spectroscopy of diffuse Galactic radiation with AKARI/IRC
}

\author{
Takashi Onaka $^{a *}$, Tamami I. Mori $^{a}$, Itsuki Sakon ${ }^{a}$, Ryou Ohsawa $^{a}$, Tomohiko \\ Nakamura, ${ }^{a}$ Ho-Gyu Lee ${ }^{a}$; Ingrid M. Koch ${ }^{b}$, Takashi Shimonishi $^{c}$, Hidehiro Kaneda $^{d}$, \\ Yoko Okada ${ }^{e}$, and Masahiro Tanaka ${ }^{f}$ \\ ${ }^{a}$ Department of Astronomy, Graduate School of Science, The University of Tokyo, Tokyo \\ 113-0033, Japan \\ ${ }^{b}$ Department of Physics and Astronomy, University of Rochester, NY 14627-0171, U. S. A. \\ ${ }^{c}$ Department of Earth and Planetary Sciences, Kobe University, Kobe 657-8501, Japan \\ ${ }^{d}$ Graduate School of Science, Nagoya University, Nagoya 464-8602, Japan \\ ${ }^{e} I$. Physikalisches Institut, Universität zu Köln, 50937 Köln, Germany \\ ${ }^{f}$ Center for Computational Sciences, Tsukuba University, Ibaraki 305-8577, Japan \\ E-mail: onaka@astron.s.u-tokyo.ac.jp
}

\begin{abstract}
We present the results of near-infrared (NIR: $2.5-5 \mu \mathrm{m}$ ) spectroscopy of diffuse Galactic sources on the Galactic plane obtained with the Infrared Camera (IRC) on board AKARI. The spectral region of $2.5-5 \mu \mathrm{m}$ is rich in various emission and absorption features. In this report, we focus on the $\mathrm{H}_{2} \mathrm{O}$ and $\mathrm{CO}_{2}$ ice absorption features at 3.0 and $4.3 \mu \mathrm{m}$, respectively, and report a search for features of deuterated polycyclic aromatic hydrocarbons (PAHs) in $4.4-4.6 \mu \mathrm{m}$. The column densities of $\mathrm{CO}_{2}$ and $\mathrm{H}_{2} \mathrm{O}$ ices show a correlation in agreement with that obtained with ISO observations. The correlation nearly crosses the origin, suggesting that $\mathrm{H}_{2} \mathrm{O}$ and $\mathrm{CO}_{2}$ ices form in tandem for a wide range of physical conditions. The ratio of the ice column densities in AFGL2591 along the slit is relatively constant over an area of $30^{\prime \prime}$, also supporting the tandem formation of $\mathrm{H}_{2} \mathrm{O}$ and $\mathrm{CO}_{2}$ ices. The $\mathrm{H}_{2} \mathrm{O}$ ice column density shows a weak trend with $A_{\mathrm{V}}$ estimated from $\mathrm{HI}$ recombination lines, which is in contrast to the clear correlation seen toward quiescent clouds. The weak correlation may be attributed partly to the uncertainty in $A_{\mathrm{V}}$ and/or a range of the environmental conditions in the present targets. Only weak excess emission is seen in $4.4-4.6 \mu \mathrm{m}$ in the spectra of the Orion bar, M17, and a reflection nebula. From these spectra, the ratio of deuterated PAHs to undeuterated PAHs is estimated as $3 \%$ at most. This is significantly smaller than the previously reported value and suggests that missing deuterium must reside in large PAHs that do not emit the $3 \mu \mathrm{m}$ bands, if it is depleted into PAHs.
\end{abstract}

The Life Cycle of Dust in the Universe: Observations, Theory, and Laboratory Experiments - LCDU 2013, 18-22 November 2013

Taipei, Taiwan

\footnotetext{
* Speaker.

${ }^{\dagger}$ The present address: Korea Astronomy and Space Science Institute, Daejeon 305-348, Republic of Korea
} 


\section{Introduction}

The near-infrared (NIR) spectral range from 2 to $5 \mu \mathrm{m}$ contains the various interesting features of gaseous and solid species in the interstellar medium (ISM). Absorption features of several major ices are observed at $3 \mu \mathrm{m}\left(\mathrm{H}_{2} \mathrm{O}\right), 4.3 \mu \mathrm{m}\left(\mathrm{CO}_{2}\right)$, and $4.6 \mu \mathrm{m}(\mathrm{CO})$ [13]. These include the fundamental vibration mode of $\mathrm{CO}$ gas, several molecular hydrogen lines, and $\mathrm{HI}$ recombination lines. In addition, this range contains the emission features of hydrocarbon dust at around $3 \mu \mathrm{m}$, which are attributed to stretching vibration modes of aromatic and aliphatic bonds in materials containing polycyclic aromatic hydrocarbons (PAHs) [6]. Deuterated hydrocarbon features are also expected to be present in $4.4-4.6 \mu \mathrm{m}$ [12]. Despite these interests, however, this spectral range has barely been explored by instruments with high sensitivity.

The Infrared Camera (IRC: [9]) onboard AKARI [7] enabled high-sensitivity spectroscopy in the NIR for the first time [8]. Even after the exhaustion of the cryogen, the IRC continued to carry out NIR observations and obtained NIR spectra in various celestial objects [10]. Here we focus on the ice absorption features and report a search for features of deuterated PAHs in the $4 \mu \mathrm{m}$ region with AKARI/IRC observations. The results of the PAH features are reported separately [6].

\section{Observations and Results}

Observations were made with the long-slit NIR grism spectroscopy mode $(R \sim 100)$ of the IRC in the warm mission phase [10]. The present data were obtained in the ISMGN [4] and IPYSO programs. The targets include PDR-HII region complexes and young stellar objects (YSOs) distributed over the Galactic plane. Among $\sim 400$ spectra, we select $\sim 90$ spectra, which show ice absorption features, and analyze them in the following. Details of the data reduction are provided in [6].

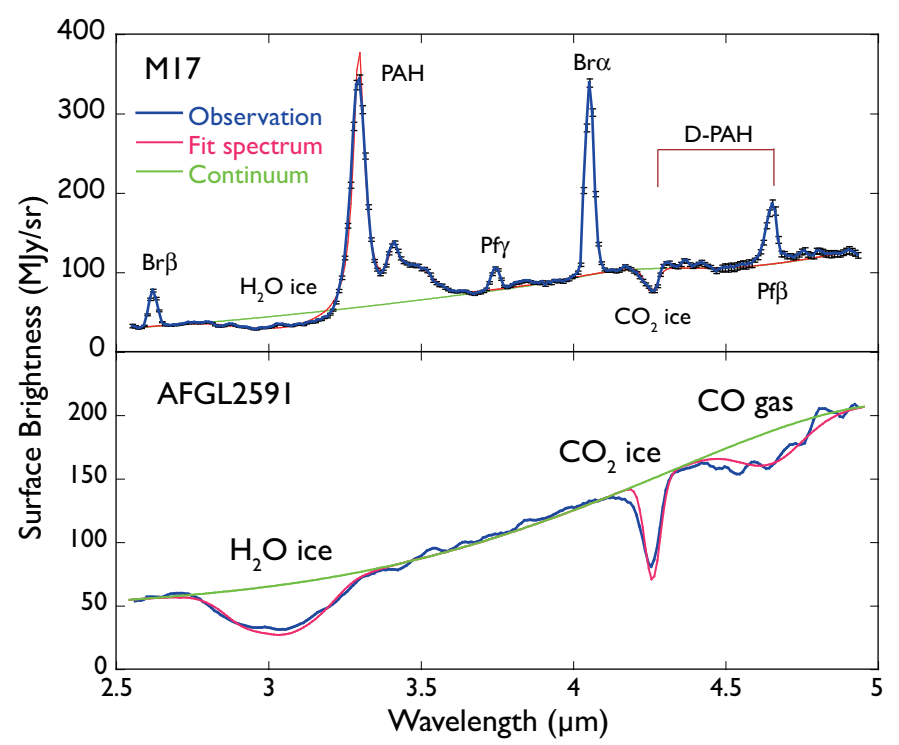

Figure 1: IRC spectra of M17 (top) and AFGL 2591 (bottom). Identifications of the features are indicated [11. The blue, red, and green lines are observed, fitted, and continuum spectra, respectively. AFGL 2591 shows a typical spectrum of a massive YSO [13]. 
Fig. 1 1 shows typical examples of spectra of the present sample. The continuum is fitted by a spline curve and then the intensities of the bands and lines are estimated. For the $\mathrm{H}_{2} \mathrm{O}$ ice feature, laboratory data at $10 \mathrm{~K}[2]$ are employed in the fit.
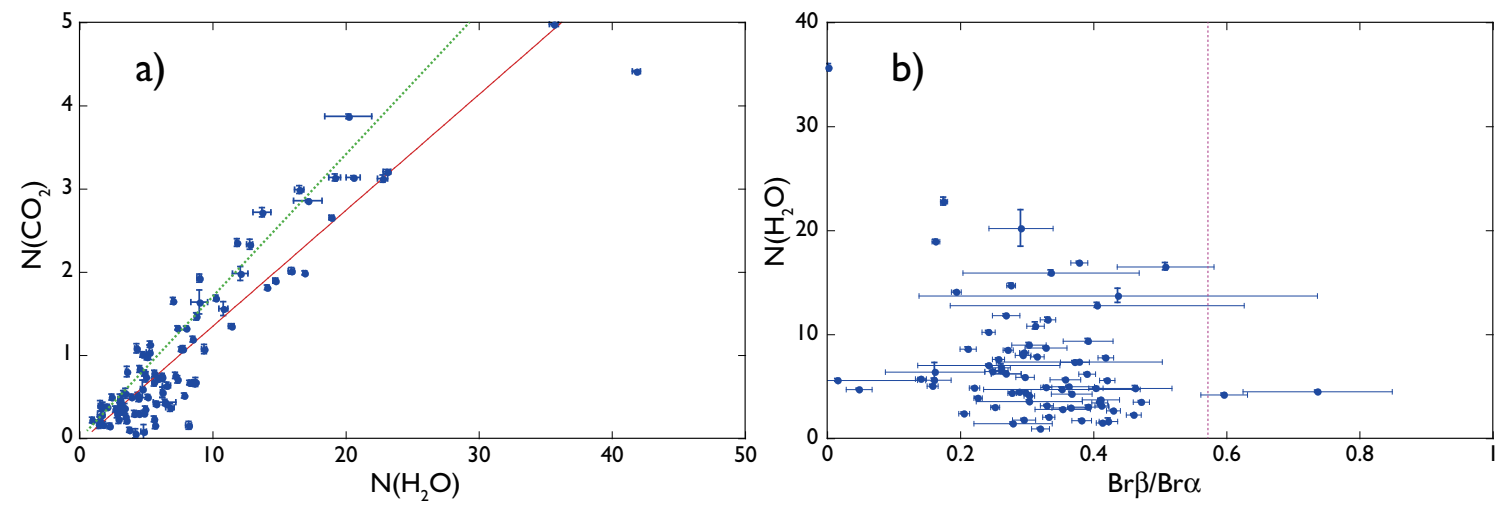

Figure 2: (a) Column density correlation between $\mathrm{H}_{2} \mathrm{O}$ and $\mathrm{CO}_{2}$ ice in units of $10^{17} \mathrm{~cm}^{-2}$. The red solid line indicates the best fit, while the green dotted line shows a correlation indicated by ISO observations [3]. Data points below the correlation line(s) can be attributed either to foreground contamination or errors in the column density estimate. (b) $\mathrm{H}_{2} \mathrm{O}$ column density vs. the line ratio of $\mathrm{Br} \beta / \mathrm{Br} \alpha$. The red vertical line indicates the ratio for the case $\mathrm{B}$ condition [14].

Fig. 2a shows the correlation between the $\mathrm{H}_{2} \mathrm{O}$ and $\mathrm{CO}_{2}$ column densities of the present sample. The best fit line gives $N\left(\mathrm{CO}_{2}\right)\left[\mathrm{cm}^{-2}\right]=(0.14 \pm 0.01) \times N\left(\mathrm{H}_{2} \mathrm{O}\right)-(0.084 \pm 0.06) \times 10^{17}$ (red line), which is in good agreement with that derived with ISO observations shown by the green line [3]. The best fit line crosses nearly the origin, suggesting that $\mathrm{H}_{2} \mathrm{O}$ and $\mathrm{CO}_{2}$ ices form in tandem for a wide range of physical conditions [15].

Fig. $2 b$ plots the $\mathrm{H}_{2} \mathrm{O}$ ice column density against the line ratio of $\mathrm{Br} \beta$ to $\mathrm{Br} \alpha$. The red vertical line indicates the line ratio for the case B conditions (all of the Lyman series transitions are optically thick) with electron density of $10^{4} \mathrm{~cm}^{-3}$ and temperature of $10^{4} \mathrm{~K}$ [14]. Only a week correlation is seen, which is in contrast to the correlation found toward quiescent clouds [15]. There seems a threshold at around $0.4-0.5$ of $\operatorname{Br} \beta / \mathrm{Br} \alpha$, above which ices are rarely present (Fig. $2 \mathrm{~b}$ ). These ratios correspond to $A_{\mathrm{V}}=4-10 \mathrm{mag}$ assuming case $\mathrm{B}$. The scatter can be attributed partly to the uncertainty in $A_{\mathrm{V}}$ and/or to a wide range of the environmental conditions of the present targets.

The spatial variation of the ice column densities is investigated in AFGL2591 along the slit over an area of $30^{\prime \prime}$. The ratio of the column densities of $\mathrm{CO}_{2}$ to $\mathrm{H}_{2} \mathrm{O}$ ices is found to be relatively constant (0.15-0.2). This result further supports the tandem formation of $\mathrm{CO}_{2}$ to $\mathrm{H}_{2} \mathrm{O}$ ices.

The fraction of deuterated PAHs is investigated based on the band ratio of $4.4-4.6 \mu \mathrm{m}$ to that in 3.3-3.5 $\mu \mathrm{m}$ in the Orion bar and M17 regions. As can be seen in Fig. 1, there is no large excess emission in the $4 \mu \mathrm{m}$ region except for $\operatorname{Pf} \beta$. The ratio of the residual emission in $4.4-4.6 \mu \mathrm{m}$ to the band intensity in $3.3-3.5 \mu \mathrm{m}$ is found to be $2-3 \%$ after subtracting the contribution from ionized gas [11]. To avoid possible contribution from remaining ionized gas component, the spectrum of the reflection nebula GN 18.14.0 is also analyzed, which indicates the band ratio of $2.1 \pm 0.2 \%$. These ratios are much smaller than the previously reported value [12], raising a question of the location of missing deuterium in the ISM [5]. If it is depleted in PAHs [1], it must reside in large PAHs that do not emit in the $3 \mu \mathrm{m}$ bands (see [11] for detailed discussion). 


\section{Acknowledgments}

This work is based on observations with AKARI, a JAXA project in participation of ESA. The authors thank all the members of the ISMGN team for their help and continuous encouragements. This work is supported in part by Grants-in-Aid for Scientific Research from the JSPS as well as by the AKARI data processing and archiving activity of ISAS/JAXA.

\section{References}

[1] Draine, B. T. Can Dust Explain Variations in the D/H Ratio?, ASP Conf. ser. , 348, 58 (2006).

[2] Ehrenfreund, P., Boogert, A. C. A., Gerakines, P. A., et al. A Laboratory Database of Solid CO and $\mathrm{CO}_{2}$ for ISO, A\&A, 315, L341 (1996).

[3] Gerakines, P. A., Whittet, D. C. B., Ehrenfreund, P., et al. Observations of Solid Carbon Dioxide in Molecular Clouds with the Infrared Space Observatory, ApJ, 522, 357 (1999).

[4] Kaneda, H., Koo, B. C., Onaka, T., \& Takahashi, H. AKARI Observations of the ISM in our Galaxy and Nearby Galaxies, Adv. Space Res., 44, 1038 (2009).

[5] Linsky, J. L., Draine, B. T., Moos, H. W., et al. What Is the Total Deuterium Abundance in the Local Galactic Disk?, ApJ, 647, 1106 (2006).

[6] Mori, T. I., Onaka, T., Sakon, I., et al. Observational Studies on the Near-Infrared Unidentified Emission Bands in Galactic HII Regions, ApJ, submitted (2014).

[7] Murakami, H., Baba, H., Barthel, P., et al. The Infrared Astronomical Mission AKARI, PASJ, 59, S369 (2007).

[8] Ohyama, Y., Onaka, T., Matsuhara, H., et al. Near-Infrared and Mid-Infrared Spectroscopy with the Infrared Camera (IRC) for AKARI, PASJ, 59, S411 (2007).

[9] Onaka, T., Matsuhara, H., Wada, T., et al. The Infrared Camera (IRC) for AKARI - Design and Imaging Performance, PASJ, 59, S401 (2007).

[10] Onaka, T., Matsuhara, H., Wada, T., et al. AKARI Warm Mission, Proc. of SPIE, 7731, 77310M (2010).

[11] Onaka, T., Mori, T. I., Sakon, I., et al. Search for the Infrared Emission Features from Deuterated Interstellar Polycyclic Aromatic Hydrocarbons, ApJ, 780, 114 (2014).

[12] Peeters, E., Allamandola, L. J., Bauschlicher, C. W., Jr., et al. Deuterated Interstellar Polycyclic Aromatic Hydrocarbons, ApJ, 604, 252 (2010).

[13] Shimonishi, T., Onaka, T., Kato, D., et al. Spectroscopic Observations of Ices around Embedded Young Stellar Objects in the Large Magellanic Cloud with AKARI, A\&A, 514, 12 (2010).

[14] Storey, P. J., \& Hammer, D. G. Recombination line intensities for hydrogenic ions-IV. Total recombination coefficients and machine-readable tables for $Z=1$ to 8, MNRAS, 272, 41 (1995).

[15] Whittet, D. C. B., Shenoy, S. S., Bergin, E. A., et al. The Abundance of Carbon Dioxide Ice in the Quiescent Intracloud Medium, ApJ, 655, 332 (2007). 